\title{
ADPIC y los Tratados Bilaterales de Inversión: la necesidad de encontrar una armonía en su aplicación
}

\author{
Gabriel M. Lentner \\ Danube University Krems \\ Sheila E. Proaño \\ Universidad San Francisco de Quito
}

\begin{abstract}
Resumen
El presente documento analiza las normas internacionales existentes sobre la protección de los derechos de propiedad intelectual en los acuerdos internacionales de inversión, con especial énfasis en los tratados bilaterales de inversión (TBI) y cómo se relaciona con el Acuerdo sobre los Aspectos de los Derechos de Propiedad Intelectual relacionados con el Comercio (ADPIC). El análisis demuestra que los estándares internacionales para la protección de los derechos de propiedad intelectual, después de la adopción del ADPIC, son inconsistentes. En el trabajo se analizan las normas ADPIC-Plus que han repercutido de manera sustancial sobre las flexibilidades previstas en el Acuerdo. En este trabajo se concluye sobre la necesidad de aclarar los parámetros jurídicos internacionales sobre el tema, y de esta manera asegurar la coexistencia armoniosa de las disposiciones acordadas en los TBI y ADPIC.
\end{abstract}

\begin{abstract}
This paper analyzes the current international standards for the protection of intellectual property rights by international investment agreements with particular emphasis on bilateral investment treaties (BITs) and how these relate to TRIPS. It demonstrates that international standards for the protection of intellectual property rights after the entry into force of the TRIPS Agreement remain inconsistent. The TRIPS-plus standards as included in BITs erode the flexibilities provided for in the TRIPS Agreement. In doing so, this paper raises the necessity of clarifying the international legal framework in this respect, in order to allow these two bodies of law to coexist in a harmonious and coherent manner.
\end{abstract}

Palabras clave: Ley de inversiones internacionales / TRIPS / TRIPS-plus / Tratados bilaterales de inversiones / Licencia obligatoria.

Key words: International Investment Law; TRIPS; TRIPS-plus; Bilateral Investment Treaties; Compulsory Licensing. 
Sumario: 1. Introducción 2. Apreciaciones generales: ADPIC, ADPIC PLUS y sus exclusiones 2.1. Antecedentes Históricos 2.2. Alcance del ADPIC 2.3. Flexibilidades en el ADPIC 2.4. ADPIC Plus 3. Inversión Extranjera Directa 3.1. Acuerdos bilaterales - ADPIC 4. Implementación jurídica del ADPIC y los TBI 4.1. Licencia Obligatoria y ADPIC 4.2. Expropiación bajo los TBI y ADPIC 4.3. Brasil vs. Merck 5. ¿Conflicto entre acuerdos normativos? $\mathbf{6}$. Conclusiones.

\section{Introducción}

En el marco del Derecho de Inversiones se defiende que la protección de la propiedad intelectual (PI) no es un fin en sí mismo. ${ }^{2}$ La garantía que se otorga a las creaciones intelectuales sirve como una herramienta para incentivar la innovación y la invención en beneficio de la sociedad. ${ }^{3}$ Para lograr este objetivo, la regulación planteada tiene que ofrecer una compensación en relación a la protección de incentivos para los actores del mercado y el acceso del público mediante la difusión de las innovaciones y creaciones. ${ }^{4}$ Este balance que la normativa debe crear, se ve reflejado mediante la limitación de los derechos exclusivos concedidos a los innovadores y creadores por un tiempo determinado. En el marco del Derecho Internacional de Inversiones ha surgido una discusión importante en cuanto al alcance de la protección que un Estado debe brindar a los inversores extranjeros.

Esta protección ha tenido una importante evolución, es así que en el año 1868 se creó la Doctrina Calvo, la cual sostiene que los Estados receptores de inversión no se encuentran en la obligación de conferir beneficios adicionales a los inversores extranjeros. ${ }^{5}$ Además dice que en caso de surgir una controversia, los inversionistas extranjeros solo podrán recurrir a los organismos de solución de conflicto que prevé el Estado receptor. ${ }^{6}$ Esta teoría fue adoptada en su mayoría por los países latinoamericanos. Sin embargo, hoy en día parece haber perdido fuerza ante el innegable posicionamiento de los tratados bilaterales de inversión (TBI), así como los tratados multilaterales.

Los documentos internacionales en materia de inversión han dado especial importancia a la protección de los derechos de propiedad intelectual, recogido en varios documentos, el presente trabajo analiza el Acuerdo sobre los Aspectos de los De-

2 Cottier, Thomas; Germann, Christophe, "TRIPS, art. 7", Concise international and European IP law: TRIPS, Paris Convention, European enforcement and transfer of technology, Cottier, Th.; Véron, P. (eds), Kluwer Law International, Virginia, 2008; Ruse-Khan, Henning Grosse, "Protecting Intellectual Property under BITs, FTAs, and TRIPS: Conflicting Regimes or Mutual Coherence?", Max Planck Institute for Intellectual Property and Competition Law Research Paper, 2011, No. 11-02, p. 3.

3 Ruse-Khan, Henning Grosse. "Protecting Intellectual Property", p. 3.

4 Ibídem

5 Shan, Wenhua, “Is Calvo Dead?", 55 The American Journal of Comparative Law, 2007, pp. 123-163.

6 Ibídem 
rechos de Propiedad Intelectual relacionados con el Comercio (ADPIC), que es considerado un documento fundamental en el tema de inversiones. Los antecedentes históricos muestran un tema que continúa siendo polémico en las relaciones políticas entre los Estados, ya que muchos países en desarrollo consideran que la implementación del ADPIC fue impuesto por sus socios comerciales económicamente más poderosos. Entre las posturas que se plantean sobre el tema se refleja que "el sistema actual se encuentra excesivamente al servicio de los intereses económicos individuales de los titulares los cuales en la mayoría de los casos son empresas multinacionales, y de esta forma se estaría amparando los intereses de países económicamente desarrollados." ${ }^{\prime 7}$ Contrario a esta postura, se expuso que la implementación de una mejor protección en el tema de propiedad intelectual en los países en desarrollo podría conducir a una mayor inversión extranjera directa (IED) ${ }^{8}$, que a su vez conllevaría al desarrollo de la innovación local. Este argumento fue impulsado por el grupo de empresarios que asistió a la Ronda de Uruguay, representando un porcentaje significativo de los inversores de IED. ${ }^{9}$ En los estudios publicados por el Banco Mundial, se refleja que mientras más altos sean los niveles de protección en temas de propiedad intelectual, mayor será la inversión extranjera ${ }^{10}$. Ante esta postura, se han presentado posiciones contrarias para determinar que las ventajas que se expusieron en las reuniones no benefician en su totalidad a ciertos países.

El Derecho debe adaptarse rápidamente a los cambios existentes, pues éste "viene condicionado por la realidad social, pre-jurídica, subyacente, al que aquél se limita a dar respuesta normativa, ordenadora de conductas, y solución a los conflictos de intereses que dicha realidad presenta". " De esta manera, el derecho de propiedad intelectual tiene que estar acorde a los cambios y evolución de la tecnología, pero sin dejar de lado la realidad social de los Estados receptores de la inversión extranjera. Se crea una necesidad por encontrar una solución para proteger los derechos envueltos en el ámbito de propiedad intelectual bajo el argumento de los altos costos incurridos en la creación de nuevas tecnologías. Así también se discute la existencia de otros instrumentos internacionales y acuerdos entre Estados sobre los derechos de propiedad intelectual, de aquí nace una dificultad al momento de interpretar las normas en cada caso.

\footnotetext{
7 Schmitz Vaccaro, Christian, "Propiedad Intelectual, Dominio Público y Equilibrio de Intereses", 36 Revista Chilena de Derecho, 2009, pp. 343-367.

8 Gervais, Daniel, “Agreement on Trade-Related Aspects of Intellectual Property Rights 1994”, Max Planck Encyclopedia of Public International Law, Wolfrum R. (ed), Oxford University Press, Oxford, 2011.

9 Ibídem

10 Ibídem

11 Rivero Hernández, Francisco, "Familia y Cambio Social: Notas Críticas”, Anuario de Derecho Civil. Núm. LIII-3, 2000, pp. 1074-1093.
} 
En el presente documento se expone la discusión en el marco del Derecho Internacional de Inversiones sobre el conflicto entre las normas del ADPIC y las estipulaciones expresadas en los Tratados Bilaterales de Inversión (TBI), surgiendo la importancia de crear un marco normativo internacional que permita la coexistencia armoniosa de estos dos instrumentos. El trabajo esta dividido en tres partes, la primera describe de manera general los puntos más sustanciales del Acuerdo y de los TBI. La segunda, expone una discusión de los efectos en la inversión extranjera directa y la tercera parte describe el conflicto que surge de los instrumentos y su impacto en los derechos de propiedad intelectual, para lo cual se analiza el caso específico de las licencias obligatorias.

\section{Apreciaciones generales: ADPIC, ADPIC PLUS y sus exclusiones}

\subsection{Antecedentes Históricos}

Los países industrializados han adoptado un rol predominante en la consecución de una normativa eficaz que proteja los derechos de propiedad intelectual. El nivel de protección de estos derechos no era unánime en todos los países, pues dependían de cada Estado para su regulación, a medida que la propiedad intelectual comenzó a tomar fuerza en el ámbito comercial, surgió una tensión importante en las relaciones económicas internacionales. ${ }^{12}$ Es así que en la década de 1980 y principios de 1990, los países desarrollados establecieron como prioridad la regulación de estos derechos. ${ }^{13}$

El esfuerzo por parte de los Estados involucrados por establecer un marco normativo culminó con la adopción de los Aspectos de los Derechos de Propiedad Intelectual relacionados con el Comercio (ADPIC), mismo que establece las normas mínimas de protección de las diversas categorías de propiedad intelectual con respecto al comercio. ${ }^{14}$ La Organización Mundial del Comercio ha categorizado este documento como el “[...] acuerdo multilateral más completo sobre propiedad intelectual."15 En el mismo, se reguló que los Estados miembros no pueden establecer protecciones inferiores a los estándares promulgados, ${ }^{16}$ así como tampoco serían obligados a

\footnotetext{
12 Organización Mundial del Comercio, "Propiedad Intelectual: protección y observancia", disponible en: https://www.wto.org/ spanish/thewto s/whatis s/tif s/agrm7 s.htm (acceso: 9/06/2015).

13 Grossman, Gene M.; Lai Edwin L-C., "International Protection of Intellectual Property”, CESIFO Working Paper, No. 790, 2002, p. 1 .

14 Organización Mundial del Comercio, "Aspectos de los derechos de propiedad intelectual relacionados con el comercio", disponible en: https://www.wto.org/spanish/docs_s/legal_s/27-trips_01_s.htm (acceso: 7/10/2015).

15 Organización Mundial del Comercio, “Acuerdo sobre los ADPIC: visión general”, disponible en: https://www.wto.org/ spanish/tratop_s/trips_s/intel2c_s.htm (acceso: 9/10/2015).

16 El Centro del Sur, ADPIC, una guía para el Sur, Ksero-Graw \& Poli- Druk Katowince, Ginebra, 1997.
} 
adoptar garantías superiores a las establecidas conforme lo menciona el artículo 1 del ADPIC. ${ }^{17}$

Es importante aclarar que el Acuerdo, que entró en vigencia el primero de enero de 1995, no es un instrumento individual, "es el Anexo 1C del Acuerdo de Marrakech por el que se establece la Organización Mundial del Comercio, firmado en Marrakech, Marruecos, el 15 de abril de 1994". ${ }^{18}$ De aquí surge la duda del por qué el documento fue adoptado dentro de un organismo relacionado con el comercio, y no fue discutido dentro de los organismos internacionales que ya habían sido fundados específicamente para el tema de propiedad intelectual, como es la Organización Mundial de la Propiedad Intelectual (OMPI), creada mediante el Acuerdo de Estocolmo en 1967. ${ }^{19}$ Existen varias hipótesis, sin embargo la más concordante con los sucesos de la época se relacionaba a la posibilidad de establecer represalias comerciales a los Estados que incumplieran con los parámetros acordados. ${ }^{20}$ Además de este tema que llama la atención, existen otros como la capacidad de negociación de los países menos desarrollados por la carencia de especialistas en ciertas áreas. ${ }^{21}$ Así también se critica la adopción de los temas que constarían en la Agenda de las reuniones, que en un principio se aprobó en Punta del Este en 1986 meramente para discutir los temas relacionados con el comercio de productos que infringían marcas o patentes; sin embargo, por presión de los países industrializados se adoptaron los temas para las negociaciones en los aspectos más amplios de la propiedad intelectual. ${ }^{22}$

\subsection{Alcance del ADPIC}

El Acuerdo sobre los ADPIC, en el marco de la Organización Mundial del Comercio (OMC), proporciona elementos normativos para la protección de los derechos de propiedad intelectual ${ }^{23}$. Al analizar el tratamiento internacional de $\mathrm{PI}$, se refleja que los tratados internacionales que regulan estos derechos establecen sólo normas mínimas para su protección ${ }^{24}$, y el Acuerdo en mención no fue la excepción. Se incluye, entre otros temas, los principios del trato de nación más favorecida y trato nacional,

17 Aspectos de los Derechos de Propiedad Intelectual Relacionados con el Comercio, 1995, Artículo 1.

18 Organización Mundial del Comercio, "Aspectos de los derechos de propiedad intelectual relacionados con el comercio", disponible en: https://www.wto.org/spanish/docs_s/legal_s/27-trips_01_s.htm (acceso: 7/10/2015).

19 Convenio que establece la Organización Mundial de la Propiedad Intelectual 1967, Artículo 1.

20 El Centro del Sur, ADPIC, una guía para el Sur, 1997, p. 7.

21 Ibídem

22 Ibídem, pp. 8-10.

23 Thomas, Cottier; Pierre Véron, "Introduction", Concise International and European IP law: TRIPS, Paris Convention, European enforcement and transfer of technology, 2008.

24 Busche, Jan, "Das TRIPs-Übereinkommen im Überblick", TRIPs: Internationales und europäisches Recht des geistigen Eigentums, Busche, J.; Stoll, P-T. (eds), Carl Heymanns Verlag, Köln, 2007, p. 15. 
en materia de derechos de propiedad intelectual "toda ventaja o privilegio que conceda un Miembro a los nacionales de cualquier otro país se otorgará inmediatamente y sin condiciones a los nacionales de todos los demás Miembros", ${ }^{25}$ de esta manera se evita la existencia de cualquier tipo de discriminación.

El instrumento multilateral, regula un sinnúmero de temas, dentro de los cuales se encuentra los derechos de autor y los derechos conexos, marcas de fábrica o de comercio, indicaciones geográficas, modelos industriales, patentes, esquemas de trazado de los circuitos integrados, incluyendo temas de competencia como es el control de las prácticas anticompetitivas en las licencias contractuales. ${ }^{26}$

El ADPIC recopila temas que ya existían en otros instrumentos internacionales como era el Convenio de París de 1883 enfocado en la regulación de temas de propiedad industrial, ${ }^{27}$ y el Convenio de Berna de 1886 encaminado a proteger obras literarias y artísticas. ${ }^{28}$ Es así que inclusive dentro de los artículos que norman los temas del ADPIC se hace referencia a estas dos convenciones:

con excepción de las disposiciones del Convenio de Berna sobre los derechos morales, todas las principales disposiciones sustantivas de esos Convenios se incorporan por referencia al Acuerdo sobre los ADPIC y se convierten así en obligaciones para los países Miembros de dicho Acuerdo. [... ] el Acuerdo sobre los ADPIC añade un número sustancial de obligaciones en aspectos que los convenios antes existentes no tratan o tratan de modo que se consideró insuficiente. Así pues, a veces se llama al Acuerdo sobre los ADPIC el Acuerdo de Berna y de París ampliado. ${ }^{29}$

Con respecto a las obligaciones de los Estados, el documento establece que los procedimientos de observancia deberán permitir la adopción de medidas eficaces ante cualquier infracción, estos procesos deben implementarse de tal manera que no se constituya en una interrupción al comercio legítimo. ${ }^{30}$ Así también se recalca la importancia de las garantías procesales, sin embargo se deja en claro que en cuanto a la observancia de los preceptos del tratado no es necesario instaurar un sistema judicial

25 Abarza, Jacqueline; Katz, Jorge, Los derechos de propiedad intelectual en el Mundo de la OMC, Santiago, 2002, disponible en: http://portal.concytec.gob.pe/images/stories/images2012/portal/areas-institucion/dag/curso-politicas-ctei/propiedad-intelectual/1. pdf (acceso: 4/10/2015).

26 Aspectos de los Derechos de Propiedad Intelectual relacionados con el Comercio (ADPIC).

27 Convenio de París, 1883.

28 Convenio de Berna para la protección de las obras literarias y artísticas, 1886.

29 Organización Mundial del Comercio, “Acuerdo sobre los ADPIC: visión general”, disponible en: https://www.wto.org/ spanish/tratop_s/trips_s/intel2_s.htm (acceso: 29/09/2015).

30 Ibídem 
distinto al existente en cada país, "ni afecta a la capacidad de los Miembros para hacer observar su legislación en general". ${ }^{31}$

Parecería que el documento cubre todos los temas importantes de la propiedad intelectual, sin embargo, se omitieron ciertos aspectos que debieron ser integrados. Entre ellos resalta la carencia de una regulación sobre el conocimiento ancestral y el folclore, se han intentado establecer parámetros para regular este tema, es así que en la Declaración de Doha se encomienda al Consejo de los ADPIC para que examine entre otras cosas "la relación entre el Acuerdo de los ADPIC y la protección de los conocimientos tradicionales y el folclore" ${ }^{32}$

La importancia de mencionado tema viene especialmente para los países en desarrollo, "las modalidades de titularidad de los conocimientos tradicionales, el interés cultural, científico, y comercial de esos conocimientos, las posibilidades de fructíferas colaboraciones en la investigación y el desarrollo, y el riesgo de utilización abusiva de esos conocimientos, no son cuestiones que se plantean únicamente dentro de las fronteras nacionales $[\ldots]^{33}$

Otro tema importante que no se incluye es el tema del agotamiento de los derechos, que también es conocida como la "doctrina de la primera venta", se refiere a que posterior de la primera venta del bien, el titular de los derechos de propiedad intelectual no se encuentra facultado para solicitar, mediante acciones de PI, la interrupción de la comercialización del producto. ${ }^{34}$ Esta primera venta debió haber sido realizada por el propietario del titular de PI, o por un tercero autorizado. ${ }^{35}$ La doctrina hace también alusión a la falta de inclusión de la protección de las obtenciones vegetales, que es recogido en el Convenio Internacional para la Protección de las Obtenciones Vegetales adoptado en París en 1961. ${ }^{36}$

De la misma manera, nada se regula acerca de los derechos morales en el campo de los derechos de autor, esto porque los países desarrollados cuyo sistema se rige por el common law no los contempla dentro de sus legislaciones. ${ }^{37}$ A pesar de haber

\footnotetext{
31 Ibídem

32 Organización Mundial de la Propiedad Intelectual, "Propiedad intelectual y conocimientos tradicionales - Folleto N ${ }^{\circ}$ ", disponible en: http://www.wipo.int/edocs/pubdocs/es/tk/920/wipo_pub_920.pdf (acceso: 11/10/2015).

33 Ibídem

34 Organización Mundial de la Propiedad Intelectual, "Seminario Regional de la OMPI para algunos países de América Latina y del Caribe sobre la implementación y el uso de ciertas flexibilidades en materia de patentes", disponible en: http://www.wipo.int/ edocs/mdocs/mdocs/en/wipo_ip_bog_12/wipo_ip_bog_12_ref_u14a_cadena.pdf (acceso: 3/10/2015).

35 Ibídem

36 Rodríguez Perozo, Nadiafna, "El acuerdo de los derechos de Propiedad intelectual relacionados con el comercio (ADPIC) y

las normas ADPIC Plus”, Revista de Derecho Escuela de postgrado, No 1,2011.

37 Ibídem
} 
faltado cierto temas, se observa la intención de materializar todos los derechos de propiedad intelectual en un solo documento internacional.

\subsection{Flexibilidades en el ADPIC}

El Acuerdo sobre los ADPIC permite ciertas exclusiones y flexibilidades para asegurar que sus obligaciones no obstaculicen indebidamente importantes objetivos de política pública ${ }^{38}$ Esto significa que los miembros de la OMC conservan un margen de actuación para la ejecución de sus obligaciones en virtud del Acuerdo..$^{39}$ Por ejemplo, el Art 27 (2) y (3), establece que los Estado miembros podrán evaluar qué temas podrían ser patentables o no, conforme al análisis de ciertos temas relacionados con el orden público, moralidad, e incluso un tema sustancial que llama la atención es la inclusión en temas de la salud o la vida de las personas o de los animales para preservar los vegetales o evitar daños al medio ambiente. ${ }^{40}$ Bajo esta premisa, el Artículo 30 menciona que los miembros de la OMC pueden crear excepciones a la concesión de derechos de patente, siempre que ello no perjudique indebidamente los intereses del titular. ${ }^{41}$ Por otra parte, el artículo 31 prevé la concesión de licencias obligatorias de patentes, sujeto a ciertas condiciones, cuando esté envuelto el "interés público" o una "emergencia nacional" ${ }^{42}$ El Artículo 40 impide las prácticas anticompetitivas que pueden impedir la transferencia de tecnología. El artículo 66 establece un período de gracia para los países menos desarrollados, durante el cual no se tienen que aplicar ciertas obligaciones de los ADPIC.

El concepto de flexibilidades de los ADPIC se ve apoyado por las reglas de interpretación del derecho internacional público codificadas en el artículo 31 de la Convención de Viena sobre el Derecho de los Tratados. Mediante el cual "un tratado deberá interpretarse de buena fe conforme al sentido corriente que haya de atribuirse a los términos del tratado en su contexto y a la luz de su objeto y fin." ${ }^{43}$ Los objetivos y principios del Acuerdo sobre los ADPIC se ven reflejados en los artículos 7 y 8, que si bien no son de carácter obligatorio, tienen una función de establecer los parámetros jurídicos del Acuerdo. Estos dos artículos son cruciales para la interpretación de las disposiciones de los ADPIC a fin de asegurar que los resultados que devienen de la

\footnotetext{
38 Smith, Van, "Enabling Environments or Enabling Discord: Intellectual Property Rights, Public-Private Partnerships, and the Quest for Green Technology Transfer", 42 Georgetown Journal of International Law, 2011, p. 832-833.

39 Ver también WTO, "Philosophy: TRIPS Attempts to Strike a Balance", disponible en: http://www.wto.org/english/tratop_e/ trips_e/factsheet_pharm01_e.htm (acceso: 10/10/2015)

40 Aspectos de los derechos de propiedad intelectual relacionados con el comercio, 1995, Artículo 27.

41 Ibídem

42 Ibídem

43 En virtud de Art.3 (2) del Entendimiento sobre Solución de Diferencias de la OMC, esta regla de interpretación de los tratados guía la interpretación de todos los acuerdos de la OMC.
} 
aplicación del documento sean favorables a los objetivos de la innovación y la difusión de información protegida por derechos de propiedad intelectual ${ }^{44}$. Esto, desafortunadamente, no ha recibido pleno apoyo en la jurisprudencia de la OMC.

Cabe señalar que estas flexibilidades se deben a la necesidad de cada país para adaptar su aplicación del Acuerdo sobre los ADPIC con miras a la integración de los objetivos nacionales de innovación y de desarrollo. Las excepciones y limitaciones no se encuentran regulados en su totalidad por los ADPIC (con excepción de las licencias obligatorias de patentes y algunos otros temas), aunque muchos están sujetos a un análisis de tres etapas. ${ }^{45}$ Esta prueba impone tres condiciones en los estados miembros de la OMC que desean introducir las excepciones y limitaciones al derecho de autor, marca registrada, diseño o derechos de patente en su legislación nacional. En esencia, esas condiciones son que la excepción o limitación debe ser (a) en casos especiales; (b) que no atente a la explotación normal de la obra u objeto protegido; y (c) que no causen un perjuicio injustificado a los intereses legítimos del titular del derecho. ${ }^{46}$ Así también, la OMC ha ampliado las exenciones existentes que permite a los Estados miembros imponer licencias obligatorias (licencias emitidas por el Estado para utilizar un derecho de propiedad intelectual), esta medida fue usada para abordar los principales problemas de salud, incluyendo las pandemias de VIH en el año $2003^{47}$.

Estas flexibilidades de los ADPIC se han destacado sobre todo en el contexto de la salud pública y los derechos humanos por parte de diversas instituciones, responsables políticos, y organizaciones no gubernamentales. ${ }^{48}$ Sin embargo, muchas de estas flexibilidades están siendo restringidas en los llamados ADPIC-Plus, acuerdos de inversión bilaterales y regionales, lo que conlleva a la existencia de un conflicto legal al momento de su aplicación.

\footnotetext{
44 Cottier, Thomas; Germann, Christophe, "TRIPS, art. 7", Concise international and European IP law: TRIPS, Paris Convention, European enforcement and transfer of technology, Cottier, Th.; Véron, P. (eds), Kluwer Law International, Virginia, 2008, pp. 29,32 .

45 Gervais, Daniel, “Agreement on Trade-Related Aspects of Intellectual Property Rights 1994”, Max Planck Encyclopedia of Public International Law, Wolfrum R. (ed), Oxford University Press, Oxford, 2011, p. 2. Para el "Test de los tres pasos" ver: Kennedy, Mathew, "The 'Three-Step Test' and the Burden of Proof in Disputes Under the TRIPS Agreement", 45 International Review of Intellectual Property and Competition Law, 2014, pp. 161-177.

46 Gervais, Daniel, “Agreement on Trade-Related Aspects of Intellectual Property Rights 1994", Max Planck Encyclopedia of Public International Law, Wolfrum R. (ed), Oxford University Press, Oxford, 2011, para. 2.

47 Ibídem

48 Ruse-Khan, Henning Grosse, "The International Law Relation between TRIPS and subsequent TRIPS-plus Free Trade Agreements: Towards safeguarding TRIPS Flexibilities?”, 18 Journal of Intellectual Property Law, 2011, p. 327.
} 


\subsection{ADPIC Plus}

El término de ADPIC Plus, ha sido recogido por la doctrina y se refiere a "todas aquellas normas o disposiciones que tienden a elevar los estándares de protección contenidos en el acuerdo sobre los ADPIC." ${ }^{49}$ La doctrina ha expuesto la forma mediante la cual se crea los ADPIC Plus, dentro de los cuales se encuentran el establecimiento de normas beneficiosas dentro de la propiedad intelectual que no se encuentran dentro del documento, también nacen de los acuerdos o cediendo al momento que se requiera una interpretación de las normas establecidas, y un tema sustancial es mediante los tratados de libre comercio bilaterales. ${ }^{50} \mathrm{~A}$ continuación analizaremos como la inclusión de estos acuerdos entre países se relaciona con la afectación o no de la inversión extranjera directa.

\section{Inversión Extranjera Directa}

\subsection{Acuerdos bilaterales - ADPIC}

Los Tratados Bilaterales de Inversión (TBI) en general protegen a inversionistas de un Estado dentro del territorio de otro. Se implementan normas que rigen el trato que el Estado receptor debe considerar en cuanto a la inversión y al establecimiento de mecanismos de solución de controversias aplicables a las presuntas violaciones de esas reglas. ${ }^{51}$ Dentro de los TBI se extienden varios derechos para el inversionista como es el derecho a un trato justo y equitativo o, inclusive el derecho a una indemnización en caso de expropiación para asegurar las inversiones..$^{52}$ Los acuerdos de inversión han sido considerados por algunos países en desarrollo como un instrumento para atraer a la inversión extranjera. ${ }^{53}$ Por lo tanto, los tratados bilaterales de inversión se encuentran dentro de las áreas de más rápido crecimiento del derecho internacional. En la actualidad existen un estimado de tres mil TBI en todo el mundo ${ }^{54}$, junto con varios acuerdos multilaterales entre Estados, incluyendo el Tratado de Libre Comercio de América del Norte (TLCAN) ${ }^{55}$, El Tratado de Libre Comercio entre Centroamérica y

\footnotetext{
49 Ibídem

50 Ibídem

51 Dolzer, Rudolf; Schreuer, Christoph, Principles of International Investment Law, Oxford University Press, Oxford, 2008.

52 Rutledge, Peter B. 'TRIPS and BITs: An Essay on Compulsory Licenses, Expropriation, and International Arbitration' 13 North Carolina Journal of Law \& Technology, 2012, p. 158.

53 Correa, Carlos M., "Bilateral Investment Agreements: Agents of new global standards for the protection of intellectual property rights?", GRAIN Reports, 2004, p. 3.

54 Un análisis pormenorizado de los tratados puede encontrarse en los documentos del Centro Internacional para Resolución de Disputas sobre Inversiones (CIADI), disponible en: https://icsid.worldbank.org/apps/ICSIDWEB/resources/Pages/BilateralInvestment-Treaties-Database.aspx (acceso: 5/10/2015).

55 Sistema de Información de Comercio Exterior. ‘Tratado de Libre Comercio de América del Norte', disponible en: http://www. sice.oas.org/trade/nafta_s/indice1.asp (acceso: 4/10/2015).
} 
los Estados Unidos (CAFTA) ${ }^{56}$, y los acuerdos comerciales firmados con la Asociación de Naciones del Sudeste Asiático (ASEAN) ${ }^{57}$, entre otros. La protección de la inversión extranjera, a través de los tratados internacionales, es considerado como uno de los éxitos jurídicos internacionales ${ }^{58}$.

Ahora bien, lo expuesto entra al tema de análisis para determinar la relación que tienen los acuerdos comerciales y de cooperación con el tema de propiedad intelectual y en el desarrollo en ciertos casos de los ADPIC Plus. A manera de ejemplo, en el primer tratado bilateral de inversión existente, suscrito entre la República Federal de Alemania y Pakistán en $1959^{59}$, ya se hizo alusión a los derechos de propiedad intelectual, en el cual se recogió una definición de la protección de la inversión donde se incluyeron "activos tales como [...] las patentes y conocimientos técnicos." "60 Aunque los acuerdos de inversión normalmente no incluyen regulaciones detalladas sobre los derechos de PI, éstos incorporan una definición amplia del término "inversión" que generalmente cubre tales derechos, de esta manera se puede concluir que los TBI generalmente protegen los derechos de propiedad intelectual como inversión protegida. ${ }^{61}$ En un estudio realizado en 119 países en desarrollo entre los años 1970 y 2001 se establece que, en efecto, la inversión extranjera directa aumenta en países que han suscrito tratados bilaterales de inversión, sin embargo no se puede dejar de lado la seguridad jurídica y el ambiente económico en cada país. ${ }^{62}$

La interpretación en cuanto al impacto positivo o negativo en relación a la inversión extranjera directa no es absoluta. Para Nogués y Finger, la implementación de normativa en el tema de propiedad intelectual no necesariamente contribuyen a una inver-

\footnotetext{
56 Comisión Económica para América Latina y el Caribe, "DR-CAFTA: aspectos relevantes seleccionados del Tratado y reformas legales que deben realizar a su entrada en vigor los países de Centroamérica y la República Dominicana”, disponible en: http://www.cepal.org/es/publicaciones/5001-dr-cafta-aspectos-relevantes-seleccionados-del-tratado-y-reformas-legales-que (acceso: 4/10/2015)

57 China Briefing. "Por qué la ASEAN es importante para su estrategia de negocios en Asia", disponible en: http://www.chinabriefing.com/news/2013/02/23/por-que-la-asean-es-importante-para-su-estrategia-de-negocios-en-asia.html\#sthash.pB8k5yL5. dpuf (acceso: 4/10/2015).

58 Simma, Bruno, "Foreign Investment Arbitration: A Place for Human Rights?", 60 International and Comparative Law Quarterly, 2011, p. 574.

59 Dolzer, Rudolf; Stevens, Margrete, Bilateral Investment Treaties, Martinus Nijhoff Publishers, The Hague, 1995, p. 267.

60 IISD, "Federal Republic of Germany and Pakistan Treaty for the Promotion and Protection of Investment", disponible en: http://www.iisd.org/pdf/2006/investment_pakistan_germany.pdf (acceso: 3/10/2015).

61 Bole, Bertram, "The Protection of Intellectual Property Rights through Bilateral Investment Treaties: Is there a TRIPS-plus Dimension?", 19 NCCR trade regulation Working Paper, 2010, p. 33. Vid. Lavery, Rachel, "Coverage of Intellectual property Rights in International Investment Agreements: An Empirical Analysis of Definitions in a Sample of Bilateral Investment Treaties and Free Trade Agreements”, 6 Transnational Dispute Management, 2009, pp. 4-7 y Annex 1.

62 Neumayer, Eric; Spess, Laura. “Do bilateral investment treaties increase foreign direct investment?", World Development, 33 (10), 2005.
} 
sión extranjera directa. ${ }^{63}$ Se analizó el caso de Argentina, donde se demostró que el crecimiento en la inversión extranjera directa fue mayor en los sectores de servicios que no estaban sujetos al ADPIC, que en aquellos en los que estaba obligado. El estudio manifestó la existencia de un incremento de cerca de 13 billones de dólares. ${ }^{64}$ Los factores clave en la atracción de inversión extranjera fueron la estabilización macroeconómica y seguridad jurídica, que vinieron amparados por los tratados bilaterales de inversión estableciendo beneficios económicos enfocados a los porcentajes de participación, remesas de capital y utilidades, condiciones de empleo. ${ }^{65}$ De la misma manera, los resultados empíricos de un estudio realizado por Smarzynska ${ }^{66}$ demuestran que la protección en la propiedad intelectual incide en las probabilidades para que exista inversión extranjera cuando se analizan los casos en el ámbito de alta tecnología, pero no en otras industrias. A pesar de estas dos tesis contrarias, hay que resaltar la importancia que el tema de IED tiene al momento de negociar y acceder a los parámetros establecidos en acuerdos internacionales.

\section{Implementación jurídica del ADPIC y los TBI}

En la doctrina clásica del Derecho se establece claramente la diferencia entre validez y eficacia de una norma, de esta manera se considera válida si fue creada conforme los preceptos jurídicos lo disponen, sin embargo, sólo será eficaz si ésta es aplicada. ${ }^{67}$ Lo que se pretende con el ADPIC es justamente aquello, lograr la eficacia de las normas establecidas a través de la armonía en la interpretación de otros instrumentos. Al ser el ADPIC un documento internacional cuyo fin fue abarcar en su mayoría los temas de propiedad intelectual, resulta complejo realizar un análisis de cada tema y su afectación, es por ello que centramos la presente sección en las licencias obligatorias que ha sido objeto de mayor estudio por la doctrina y que ayudará a esclarecer si el ADPIC y los TBI pueden coexistir en el campo jurídico.

\subsection{Licencia Obligatoria y ADPIC}

Como hemos expuesto en secciones anteriores, la licencia obligatoria es una de las flexibilidades que permite el ADPIC $^{68}$ aplicable a patentes y se configura cuando las

\footnotetext{
63 Finger, J. M.; Nogués, Julio J., The Unbalanced Uruguay Round Outcome, World Bank Publications, 2001. 64 Ibídem 65 Ibídem

66 Smarzynska, Beata K. "Composition of Foreign Direct Investment and Protection of Intellectual Property Rights: Evidence from Transition Economies", disponible en: http://www.iatp.org/files/Composition_of_Foreign_Direct_Investment_and_P.htm 67 Kelsen, Hans, "Validez y eficacia de la norma", disponible en: http://biblio.juridicas.unam.mx/libros/4/1956/10.pdf (acceso: $2 / 10 / 2015)$ 68 Aspectos de los derechos de propiedad intelectual relacionados con el comercio (1995). Artículo 31.
} 
autoridades de un país conceden una licencia a terceros diferentes del titular de la patente $^{69}$. De esta manera, se puede usar los derechos otorgados que incluyen el fabricar, usar, vender, o importar un producto patentado o un producto fabricado mediante un proceso patentado, sin el permiso de su titular. ${ }^{70}$ "Desde enero de 1995, fecha de la entrada en vigor general del Acuerdo sobre los ADPIC, al menos 12 países en desarrollo y países menos adelantados (PMA) han concedido licencias obligatorias o han determinado el uso público no comercial [... ] de las patentes" ${ }^{71}$ El uso de éstas licencias se ha visto aplicadas en su vasta mayoría en temas de fármacos. La doctrina ha sido exhaustiva al momento de relacionar el ADPIC con el derecho de salud, puesto que se ha considerado que:

[...] los medicamentos desempeñan un papel social de importancia, en la medida en que son parte integral del logro de un derecho humano fundamental, el derecho a la salud. De ahí que se clasifiquen como bienes esenciales, para subrayar que deben ser accesibles para todas las personas. ${ }^{72}$

El tema ha sido recogido por diversas corrientes de la doctrina, ${ }^{73}$ argumentando la limitación a los derechos de propiedad intelectual por razones de salud pública, por lo que no es necesario profundizar en las razones expuestas en el documento. En lo que respecta a nuestro trabajo, presentamos la discusión del tratamiento que la otorgación de las licencias obligatorias tiene en el marco de los acuerdos de inversión bajo el ámbito de los TBI.

\subsection{Expropiación bajo los TBI y ADPIC}

Las disposiciones sobre los derechos de propiedad intelectual en los TBI establecen la aplicabilidad de la norma de expropiación ${ }^{74}$. Esto tiene un impacto significativo sobre el funcionamiento del derecho de propiedad intelectual, puesto que a través de una licencia obligatoria, la autoridad gubernamental interfiere directamente en una patente de propiedad privada, para autorizar su uso por el gobierno o por terceras

69 Organización Mundial del Comercio, "Glosario de Términos", disponible en: https://www.wto.org/spanish/thewto_s/ glossary_s/licencia_obligatoria_s.htm (acceso: 3/10/2015)

70 Ibídem

71 El Centro del Sur, "El Uso de Licencias Obligatorias en América Latina", disponible en: http://es.southcentre.int/question/ el-uso-de-licencias-obligatorias-en-america-latina/ (acceso: 3/10/2015)

72 Organización Mundial de la Salud, "Globalización y acceso a los medicamentos. Perspectivas sobre el Acuerdo ADPIC/ OMC", Serie Economía de la salud y medicamentos, No.07, segunda edición.

73 Ver también Silva, Alberto Cerda, "Licencias obligatorias por razones de salud pública en Chile. Un análisis comparativo con el acuerdo sobre el Adpic", 16 Revista lus et Praxis, 2010, pp. 331-352.

74 Ruse-Khan, Henning Grosse, "Protecting Intellectual Property under BITs, FTAs, and TRIPS: Conflicting Regimes or Mutual Coherence?", 11 Max Planck Institute for Intellectual Property and Competition Law Research Paper, 2011, p. 3. 
partes, bajo ciertos términos ${ }^{75}$. En particular, la noción amplia y basada en efectos de expropiación indirecta en relación a la protección de la inversión internacional, permite a los titulares de derechos de propiedad intelectual extranjeros la posibilidad de impugnar las medidas estatales que limitan la explotación comercial de sus bienes o servicios protegidos mediante derechos de propiedad intelectual ${ }^{76}$. Esto también podría afectar a otras excepciones y limitaciones a estos derechos, tales como "derechos negativos" y el principio de agotamiento del derecho, los cuales son límites de protección de PI aceptados internacionalmente. ${ }^{77}$ Surge una discusión basada en si las disposiciones de expropiación en virtud de acuerdos internacionales de inversión bilaterales restringen de alguna manera la emisión de licencias obligatorias o revocación de patentes; también se discute si éstas interfieren con la protección de los intereses públicos, como la salud. ${ }^{78}$ Este elemento es sustancial en el análisis puesto que el ADPIC "consagra en el derecho internacional público el derecho de los gobiernos a adoptar diversos tipos de medidas que matizan o limitan los derechos de propiedad intelectual, incluso por motivos de salud pública,"79 el análisis se presenta a continuación.

Las preguntas que nos planteamos se enfocan en ¿cuál es la relación entre las licencias obligatorias en virtud de los ADPIC y la protección contra la expropiación bajo los TBI? ¿el otorgamiento de una licencia obligatoria, bajo los preceptos del ADPIC no se considera una expropiación indirecta bajo un TBI? Por otro lado, ¿si una licencia obligatoria no es compatible con los ADPIC, es automáticamente una expropiación?

Debido al amplio significado de lo que llamamos "expropiación indirecta", resulta difícil un análisis más preciso del impacto que esta norma tiene sobre la flexibilidades de los ADPIC. ${ }^{80}$ En general, la expropiación indirecta o "de facto" tiene lugar cuando las medidas expropiatorias, aunque no se ejecuta como una confiscación directa de la propiedad, tienen el efecto equivalente de la misma, privar al dueño de los beneficios sustanciales de su propiedad. ${ }^{81}$ Para responder a los cuestionamientos planteados

75 Gibson, Christopher, “A Look at the Compulsory License in Investment Arbitration: The Case of Indirect Expropriation”, 25 American University International Law Review, 2010, p. 361.

76 Ruse-Khan, Henning Grosse, "Protecting Intellectual Property", p. 3.

77 Ibídem

78 Liberti, Lahra, "Intellectual Property Rights in International Investment Agreements: An overview", 1 OECD Working Papers on International Investment, 2010, p. 6.

79 Organización Mundial del Comercial. Explicación de la declaración de Doha relative a los ADPIC. https://www.wto.org/ spanish/tratop_s/trips_s/healthdeclexpln_s.htm (acceso: 23/04/2015)

80 Ruse-Khan, Henning Grosse, "Protecting Intellectual Property", p. 3.

81 Ruzza, Alice, "Expropriation and Nationalization”, Max Planck Encyclopedia of Public International Law, Wolfrum, R. (ed), Oxford University Press, Oxford, 2013, para. 13; vid. Hoffmann, Anne K., "Indirect Expropriation”, Standards of Investment Protection, Reinisch, A. (ed), Oxford University Press, Oxford, 2008, pp. 151-171; Reinisch, August, "Expropriation”, The Oxford 
tomamos como fuente de análisis el Modelo de Tratado Bilateral de expropiación recogido por los Estados Unidos, cuyo artículo 6 establece que: ${ }^{82}$

Ninguna Parte expropiará ni nacionalizará una inversión, sea directa o indirectamente, mediante medidas equivalentes a la expropiación o nacionalización ("expropiación "), salvo: (a) por causa de utilidad pública; (b) de una manera no discriminatoria; (c) mediante el pago de una indemnización pronta, adecuada y efectiva; y (d), de conformidad con el debido proceso de la ley y en base a lo recogido en el artículo 5 (1), (2) y (3) “[traducción propia]

La segunda situación abordada por el Artículo 6 [Expropiación e indemnización] (1) es la expropiación indirecta, cuando un recurso o una serie de actos tienen un efecto equivalente a la expropiación directa sin la transferencia formal del título o confiscación directa.

(a) La determinación de si un acto o una serie de actos de una Parte, en una situación de hecho específica, constituye una expropiación indirecta, requiere de una investigación caso por caso basado en hechos que considere entre otros factores: (i) el impacto económico de la acción gubernamental, aunque el hecho de que una acción o una serie de actos de una Parte tenga un efecto adverso sobre el valor económico de una inversión, por sí solo, no establece que se ha producido una expropiación indirecta; (ii) el grado en que la acción del gobierno interfiere con expectativas de inversión inequívocas y razonables; y (iii) el carácter de la acción gubernamental ${ }^{83}$.

(b) Salvo en circunstancias excepcionales, los actos regulatorios no discriminatorios de una Parte que son diseñados y aplicados para proteger objetivos legítimos de bienestar público, como la salud pública, la seguridad y el medio ambiente, no constituyen expropiaciones indirectas ${ }^{84}$.

De esta manera demostramos el alcance y la magnitud del impacto en la ejecución de las flexibilidades de los ADPIC bajo la esfera del valor económico de la patente. ${ }^{85}$ En cuanto a las licencias obligatorias, el cumplimiento de la obligación en virtud del artículo 31 sobre los ADPIC para proporcionar una compensación económica adecuada,

\footnotetext{
Handbook of International Investment Law, Muchlinski, P; Ortino, F; Schreuer, Ch. (eds), Oxford University Press, Oxford, 2008, pp. $421 \mathrm{ff}$.

82 US Model of Bilateral Treaty, disponible en: https:/ustr.gov/sites/default/files/BIT\%20text\%20for\%20ACIEP\%20Meeting. pdf (acceso: 10/10/2015)

83 Ibídem

84 Ibídem

85 Ruse-Khan, Henning Grosse, "Protecting Intellectual Property”, p. 3.
} 
en la mayoría de casos excluye el valor económico real de la patente ${ }^{86}$, perjudicando así a su titular. Ahora bien es importante aclarar si el otorgamiento de una licencia obligatoria puede ser considerada como expropiación, el punto se analiza conforme al estudio de un caso concreto.

\subsection{Brasil vs. Merck}

Para el año 2000, los datos del Banco Mundial reflejaban que mas de un millón de personas tendrían VIH en Brasil. ${ }^{87}$ Es así que el Estado se vio en la necesidad de tomar medidas dentro de las cuales se establecía la subvención de estos medicamentos a precios accesibles al público, esto conllevó a la negociación con la empresa de farmacéuticos Merck. En vista de que las negociaciones no resultaron fructuosas a los intereses del país, el gobierno usó la estrategia de la licencia obligatoria. En cuanto a nuestro análisis respecta, llama la atención el argumento usado por la farmacéutica, al establecer que existió una expropiación de propiedad intelectual. ${ }^{88}$ Para doctrinarios como Feldman, ${ }^{89}$ la discreción permitida en cuanto a la aplicación de las norma del ADPIC en el caso mencionado es preocupante, debido a que previo a que se expida la licencia obligatoria, la farmacéutica había acordado reducir su precio en un 30\%, propuesta que no fue aceptada por el Estado..$^{90}$ Ahora bien, este caso tuvo un efecto en el TBI, debido a que Estados Unidos colocó a Brasil dentro de la Lista de Vigilancia Prioritaria, ${ }^{91}$ el efecto que esto causa en las inversiones es el posible aumento de impuestos en las tarifas de exportación. ${ }^{92}$

Al estar expresamente autorizado en el Acuerdo, la interpretación expresa es que el otorgamiento de la licencias obligatorias bajo los acuerdos del ADPIC no se considera una expropiación. Aunque para una parte de la doctrina, en ciertos casos sería más adecuado configurarle como tal porque bajo esta premisa se podría demandar directamente al Estado. ${ }^{93}$ Por otro lado, las licencias obligatorias, bajo el argumento de interés público cuando no tiene un origen ilícito o discriminatorio no tiene mayor

\footnotetext{
86 Correa, Carlos M., "Bilateral Investment Agreements", p. 3.

87 Rutledge, Peter R. "Trips and Bits: an essay on compulsory licenses, expropriation, and international arbitration”, disponible en: http://digitalcommons.law.uga.edu/cgi/viewcontent.cgi?article=1825\&context=fac_artchop (acceso: 2/10/2015) 88 Ibídem

89 Feldman, Jamie, "Compulsory License: The Dangers Behind the Current Practice", Journal of International Business and Law, Volume 8, Issue 1, 2009.

90 Ibídem

91 Matthews, Jon, "Renewing Healthy Competition: Compulsory Licenses and Why Abuses of the TRIPS Article 31 Standards Are Most Damaging to the United States Healthcare Industry", The Journal of Business, Entrepreneurship \& the Law, Volume 4, Issue 1, 2010.

92 Ibídem

93 Correa, Carlos M., "Bilateral Investment Agreements", p. 3.
} 
lugar para una defensa. ${ }^{94}$ Del presente caso resalta el conflicto que existe entre los instrumentos de inversión, por un lado Brasil actúa conforme lo prevé el Acuerdo del ADPIC, y por otro lado, Estados Unidos toma prevalencia en los tratados bilaterales de inversión.

\section{5. ¿Conflicto entre acuerdos normativos?}

El análisis presentado demuestra las diferencias existentes en las normas de protección de los DPI en los acuerdos de inversión y los ADPIC en el tema de las licencias obligatorias. Cabe analizar como estos dos instrumentos pueden coexistir bajo conceptos de armonía y coherencia. ${ }^{95}$ Es evidente que la relación entre los TBI, relacionados con los acuerdos de libre comercio y los ADPIC se rigen por el derecho internacional. Es así que al existir conflicto entre las normas se requiere una solución mediante el principio de la interpretación armoniosa de los instrumentos, así como de una integración sistémica de los preceptos. ${ }^{96}$ Bajo los principios de interpretación del Convenio de Viena se establece la presunción contra el conflicto:

hay que interpretar los acuerdos de la OMC en su conjunto, para evitar el conflicto [... ], también hay que interpretar el derecho de la OMC de una manera compatible con el derecho público internacional cuando sea posible. Cuando hay dos posibles interpretaciones de un término, se prefiere la interpretación que evite el conflicto con otras normas. ${ }^{97}$

Esto significa que las normas de protección de inversiones y de los ADPIC se interpretarán de una forma que obvie los conflictos normativos entre los dos tratados. En cuanto a la prohibición de la expropiación en los tratados de inversión, se debe dejar espacio suficiente para una interpretación armoniosa, pues deberá tomarse en cuenta las flexibilidades del ADPIC con respecto a las licencias obligatorias (artículo 31 (3) (c) Convención de Viena). Además no hay ninguna norma en el ADPIC que prohíba a los miembros de la OMC a suscribir acuerdos bilaterales de inversión o suscribir capítulos de inversión dentro de los tratados de libre comercio. En vista del

\footnotetext{
94 Ibídem

95 Ruse-Khan, Henning Grosse, "Protecting Intellectual Property", p. 3.

96 Ver el caso sobre petróleo (Iran v United States of America) 42 ILM 1334 (2003), para 41; Mclachlan, Campbell, "The Principle of Systemic Integration and Article 31(1)(c) of the Vienna Convention", 54 International and Comparative Law Quarterly, 2005, p. 279; International Law Commission, Conclusions of the work of the Study Group on the Fragmentation of International Law: Difficulties arising from the Diversification and Expansion of International Law, United Nations, 2006, p. 7; Pitea, Cesare, "Interpreting the ECHR in the Light of 'Other' International Instruments: Systemic Integration or Fragmentation of Rules on Treaty Interpretation?", International Courts and the Development of International Law. Essays in Honour of Tullio Treves, Boschiero, N. et alia (eds), Springer, New York, 2013, pp. 545-559.

97 Condon, Bradly J., El Derecho de la Organización Mundial del Comercio. Tratados Jurisprudencia y Práctica. Cameron May, Londres, 2007, p. 56.
} 
surgimiento de estos conflictos que se han dado por las flexibilidades que permite el ADPIC, varios países han optado por tomar medidas al respecto.

Si bien los acuerdos de inversión no han sido decisivos en atraer la IED, ellos se han convertido en una fuente de demandas arbitrales multimillonarias. En particular, el derecho de los inversores a iniciar acciones directamente contra los gobiernos de los países donde se realizó la inversión genera dificultades sin precedentes para la adopción de diversas políticas públicas por parte de los gobiernos. En vista de las implicancias de los TBI y otros acuerdos de inversión, el Ecuador decidió denunciar todos los TBI que había firmado. Por su parte, Sudáfrica decidió no firmar ningún TBI nuevo e intentará renegociar o salirse de los que están vigentes. Asimismo, Australia anunció que no aceptará disposiciones de resolución directa de conflictos entre el Estado y los inversores en los nuevos acuerdos de inversión, y la India está revisando los TBI de los que es parte, especialmente las disposiciones relativas a la resolución de conflictos. ${ }^{98}$

Las medidas que han tomado ciertos gobiernos es una respuesta al intento de países desarrollados de bloquear las flexibilidades que permite el ADPIC, e instaurar a su favor nuevos parámetros de protección a través de los TBI.

\section{Conclusiones}

El presente trabajo ha analizado las normas internacionales para la protección de los derechos de propiedad intelectual en los acuerdos internacionales de inversión, con especial énfasis en los tratados bilaterales de inversión y cómo se relacionan con los ADPIC. Se ha demostrado que las normas internacionales para la protección de los derechos de propiedad intelectual después de la entrada en vigor del Acuerdo sobre los ADPIC siguen siendo incompatibles. ${ }^{99}$ Los TBI y otros acuerdos de inversión parecen ser utilizado por las naciones desarrolladas como un vehículo para elevar las denominadas "normas mínimas" de los ADPIC. ${ }^{100}$ En lugar de actuar como un techo, como se ha esperado por muchos en los países en desarrollo, el instrumento multila-

\footnotetext{
98 Correa, Carlos M., "Los acuerdos de inversión: ¿una nueva amenaza para las flexibilidades previstas en el acuerdo sobre los ADPIC?", disponible en: http://es.southcentre.int/question/los-acuerdos-de-inversion-una-nueva-amenaza-para-las-flexibilidadesprevistas-en-el-acuerdo-sobre-los-adpic/ (acceso: 25/04/2016).

99 Anderson, Alan M.; Razavi Bobak , "The Globalization of Intellectual Property Rights: TRIPS, BITs, and the Search for Uniform Protection", 38 Georgia Journal of International and Comparative Law, 2011, pp. 265-291.

100 Barbosa, Denis Borges; Chon, Margaret; von Hase, Andres Moncayo, "Symposium: The International Intellectual Property Regime Complex: Slouching towards Development in International Intellectual Property", 71 Michigan State Law Review, 2007, pp. 87-88.
} 
teral de los ADPIC se presenta como un instrumento con normas mínimas que obliga a los Estados a acatar protecciones más fuertes en este tema. Es así que, en efecto, las normas ADPIC-plus menoscaban las flexibilidades en el Acuerdo sobre los ADPIC como se ha demostrado con el caso de las licencias obligatorias. También parece que los países desarrollados continuarán usando los TBI y otros acuerdos de inversión como un medio para aumentar la protección de las normas mínimas de los derechos de propiedad intelectual contenidas en el ADPIC. Sin embargo, también han surgido acciones por parte de los países menos desarrollados para denunciar los TBI, o establecer políticas para no aceptar futuros acuerdos de ese tipo.

En conclusión, el régimen de protección internacional de los derechos de propiedad intelectual aún requiere la implementación de normas sustantivas que aseguren una armonía al momento de su aplicación. ${ }^{101}$ 


\section{Bibliografía}

Abarza, Jacqueline; Katz, Jorge, Los derechos de propiedad intelectual en el Mundo de la OMC, CEPAL, Santiago, 2002 disponible en: http://portal.concytec.gob.pe/ images/stories/images2012/portal/areas-institucion/dag/curso-politicas-ctei/ propiedad-intelectual/1.pdf (acceso: 4/10/2015)

Anderson, Alan M.; Razavi Bobak, "The Globalization of Intellectual Property Rights: TRIPS, BITs, and the Search for Uniform Protection", 38 Georgia Journal of International and Comparative Law, 2011.

Barbosa, Denis Borges; Chon, Margaret; von Hase, Andres Moncayo, "Symposium: The International Intellectual Property Regime Complex: Slouching towards Development in International Intellectual Property", 71 Michigan State Law Review, 2007.

Bole, Bertram, "The Protection of Intellectual Property Rights through Bilateral Investment Treaties: Is there a TRIPS-plus Dimension?", 19 NCCR trade regulation Working Paper, 2010.

Busche, Jan, "Das TRIPs-Übereinkommen im Überblick", TRIPs: Internationales und europäisches Recht des geistigen Eigentums, J. Busche ; P-T Stoll (eds.), Carl Heymanns Verlag, Köln, 2007.

Condon, Bradly J., El Derecho de la Organización Mundial del Comercio. Tratados Jurisprudencia y Práctica, Cameron May, Londres, 2007.

Correa, Carlos M., "Bilateral Investment Agreements: Agents of new global standards for the protection of intellectual property rights?", GRAIN Reports, 2004

Cottier, Thomas; Germann, Christophe, "TRIPS, art. 7", Concise international and European IP law: TRIPS, Paris Convention, European enforcement and transfer of technology, Th. Cottier, ; P. Véron (eds.), Kluwer Law International, Virginia, 2008 .

Cottier, Thomas; Verón, Pierre, "Introduction", Concise International and European IP law: TRIPS, Paris Convention, European enforcement and transfer of technology, 2008.

Dolzer, Rudolf; Schreuer, Christoph, Principles of International Investment Law. Oxford University Press, Oxford, 2008. 
Dolzer, Rudolf; Stevens, Margrete, Bilateral Investment Treaties, Martinus Nijhoff Publishers, The Hague, 1995.

El Centro del Sur, "El Uso de Licencias Obligatorias en América Latina”, disponible en: http://es.southcentre.int/question/el-uso-de-licencias-obligatorias-enamerica-latina/ (acceso: 3/10/2015).

Feldman, Jamie, "Compulsory License: The Dangers Behind the Current Practice", Journal of International Business and Law, Volume 8, Issue 1, 2009.

Finger, J. M.; Nogués, Julio J., The Unbalanced Uruguay Round Outcome, World Bank Publications, 2001

Gervais, Daniel, "Agreement on Trade-Related Aspects of Intellectual Property Rights 1994", Max Planck Encyclopedia of Public International Law, Wolfrum R. (ed.), Oxford University Press, Oxford, 2011.

Gibson, Christopher, "A Look at the Compulsory License in Investment Arbitration: The Case of Indirect Expropriation", 25 American University International Law Review, 2010.

Grossman, Gene M.; Lai Edwin L-C, "International Protection of Intellectual Property", CESIFO Working Paper, 2002.

Hoffmann, Anne K., "Indirect Expropriation”, Standards of Investment Protection, Reinisch, A. (ed), Oxford University Press, Oxford, 2008

Kelsen, Hans, "Validez y eficacia de la norma”, disponible en: http://biblio.juridicas. unam.mx/libros/4/1956/10.pdf (acceso: 2/10/2015)

Kennedy, Matthew, “The 'Three-Step Test' and the Burden of Proof in Disputes Under the TRIPS Agreement2, 45 International Review of Intellectual Property and Competition Law, 2014.

Lavery, Rachel, "Coverage of Intellectual property Rights in International Investment Agreements: An Empirical Analysis of Definitions in a Sample of Bilateral Investment Treaties and Free Trade Agreements", 6 Transnational Dispute Management, 2009

Liberti, Lahra, "Intellectual Property Rights in International Investment Agreements: An overview", 1 OECD Working Papers on International Investment, 2010.

Matthews, Jon, “Renewing Healthy Competition: Compulsory Licenses and Why Abu- 
ses of the TRIPS Article 31 Standards Are Most Damaging to the United States Healthcare Industry", The Journal of Business, Entrepreneurship $\varepsilon$ the Law, Volume 4, Issue 1, 2010.

Mclachlan, Campbell, "The Principle of Systemic Integration and Article 31(1)(c) of the Vienna Convention", 54 International and Comparative Law Quarterly, 2005.

Neumayer, Eric; Spess, Laura, “Do bilateral investment treaties increase foreign direct investment?", World Development, 33 (10), 2005.

Pitea, Cesare, "Interpreting the ECHR in the Light of 'Other' International Instruments: Systemic Integration or Fragmentation of Rules on Treaty Interpretation?", International Courts and the Development of International Law. Essays in Honour of Tullio Treves, Boschiero, N. et alia (eds), Springer, New York, 2013.

Reinisch, August, "Expropriation", The Oxford Handbook of International Investment Law, Muchlinski, P; Ortino, F; Schreuer, Ch. (eds), Oxford University Press, Oxford, 2008.

Rivero Hernández, Francisco, "Familia y Cambio Social: Notas Críticas", Anuario de Derecho Civil. Núm. LIII-3, 2000.

Rodríguez Perozo, Nadiafna, "El acuerdo de los derechos de Propiedad intelectual relacionados con el comercio (ADPIC) y las normas ADPIC Plus", Revista de Derecho Escuela de postgrado, No 1, 2011.

Ruse-Khan, Henning Grosse, "Protecting Intellectual Property under BITs, FTAs, and TRIPS: Conflicting Regimes or Mutual Coherence?", Max Planck Institute for Intellectual Property and Competition Law Research Paper, 2011, No. 11-02.

Ruse-Khan, Henning Grosse, "The International Law Relation between TRIPS and subsequent TRIPS-plus Free Trade Agreements: Towards safeguarding TRIPS Flexibilities?", 18 Journal of Intellectual Property Law, 2011.

Rutledge, Peter B., "TRIPS and BITs: An Essay on Compulsory Licenses, Expropriation, and International Arbitration", 13 North Carolina Journal of Law \& Technology, 2012

Ruzza, Alice, “Expropriation and Nationalization”, Max Planck Encyclopedia of Public International Law, Wolfrum, R. (ed), Oxford University Press, Oxford, 2013

Schmitz Vaccaro, Christian, "Propiedad Intelectual, Dominio Público y Equilibrio de 
Intereses", 36 Revista Chilena de Derecho, 2009.

Shan, Wenhua, "Is Calvo Dead?", 55 The American Journal of Comparative Law, 2007.

Simma, Bruno, "Foreign Investment Arbitration: A Place for Human Rights?", 60 International and Comparative Law Quarterly, 2011.

Smarzynska, Beata K., "Composition of Foreign Direct Investment and Protection of Intellectual Property Rights: Evidence from Transition Economies", disponible en: http://www.iatp.org/files/Composition_of_Foreign_Direct_Investment_and_P.htm_(acceso: 2/10/2015)

Smith, Van, "Enabling Environments or Enabling Discord: Intellectual Property Rights, Public-Private Partnerships, and the Quest for Green Technology Transfer", 42 Georgetown Journal of International Law, 2011.

\section{Legislación, tratados, resoluciones y sentencias}

Centro Internacional para Resolución de Disputas sobre Inversiones (CIADI), disponible en: https://icsid.worldbank.org/apps/ICSIDWEB/resources/Pages/Bilateral-Investment-Treaties-Database.aspx (acceso: 5/10/2015)

China Briefing. "Por qué la ASEAN es importante para su estrategia de negocios en Asia", disponible en: http://www.china-briefing.com/news/2013/02/23/ por-que-la-asean-es-importante-para-su-estrategia-de-negocios-en-asia. html\#sthash.pB8k5yL5.dpuf (acceso: 4/10/2015)

Comisión Económica para América Latina y el Caribe. "DR-CAFTA: aspectos relevantes seleccionados del Tratado y reformas legales que deben realizar a su entrada en vigor los países de Centroamérica y la República Dominicana", disponible en: http://www.cepal.org/es/publicaciones/5001-dr-cafta-aspectos-relevantes-seleccionados-del-tratado-y-reformas-legales-que (acceso: $4 / 10 / 2015)$

Corte Internacional de Justicia, "Irán vs. Estados Unidos de América”, 42 ILM 1334, 2003.

IISD, "Federal Republic of Germany and Pakistan Treaty for the Promotion and Protection of Investment", disponible en: http://www.iisd.org/pdf/2006/investment_ pakistan_germany.pdf (acceso: 3/10/2015)

Organización Mundial de la Propiedad Intelectual. "Propiedad intelectual y conoci- 
mientos tradicionales - Folleto $\mathrm{N}^{\circ} 2$ ", disponible en: http://www.wipo.int/ edocs/pubdocs/es/tk/920/wipo_pub_920.pdf (acceso: 3/10/2015).

Organización Mundial de la Propiedad Intelectual. "Seminario Regional de la OMPI para algunos países de América Latina y del Caribe sobre la implementación y el uso de ciertas flexibilidades en materia de patentes", disponible en: http://www.wipo.int/edocs/mdocs/mdocs/en/wipo_ip_bog_12/wipo_ip_ bog_12_ref_ul4a_cadena.pdf (acceso: 3/10/2015).

Organización Mundial de la Salud. Globalización y acceso a los medicamentos. Perspectivas sobre el Acuerdo ADPIC/OMC - Serie "Economía de la salud y medicamentos", No. 07, segunda edición.

Organización Mundial del Comercial. Explicación de la declaración de Doha relative a los ADPIC. https://www.wto.org/spanish/tratop_s/trips_s/healthdeclexpln_s. htm (acceso: 23/04/2015).

Organización Mundial del Comercio, "Propiedad Intelectual: protección y observancia", disponible en: https://www.wto.org/spanish/thewto_s/whatis_s/tif_s/ agrm7_s.htm (acceso: 9/06/2015)

Organización Mundial del Comercio, "Acuerdo sobre los ADPIC: visión general", disponible en: https://www.wto.org/spanish/tratop_s/trips_s/intel2c_s.htm (acceso: 9/10/2015)

Organización Mundial del Comercio, "Aspectos de los derechos de propiedad intelectual relacionados con el comercio", disponible en: https://www.wto.org/ spanish/docs_s/legal_s/27-trips_01_s.htm (acceso: 7/10/2015).

Organización Mundial del Comercio. "Glosario de Términos", disponible en: https:// www.wto.org/spanish/thewto_s/glossary_s/licencia_obligatoria_s.htm (acceso: $3 / 10 / 2015)$.

Sistema de Información de Comercio Exterior. "Tratado de Libre Comercio de América del Norte", disponible en: http://www.sice.oas.org/trade/nafta_s/indicel.asp (acceso: 4/10/2015). 